Article

\title{
Platyphylloside Isolated from Betula platyphylla is Antiproliferative and Induces Apoptosis in Colon Cancer and Leukemic Cells
}

\author{
Joo-Eun Lee ${ }^{1, \dagger}$, Nguyen Thi Thanh Thuy ${ }^{2, \dagger}$, Jina Lee ${ }^{3}$, Namki Cho ${ }^{2, *}$ and Hee Min Yoo ${ }^{3, *}$ (D) \\ 1 Stem Cell Research Center, Korea Research Institute of Bioscience and Biotechnology (KRIBB), \\ Daejeon 34141, Korea ${ }^{2}$ College of Pharmacy, Chonnam National University, Gwangju 61186, Korea \\ 3 Center for Bioanalysis, Korea Research Institute of Standards and Science (KRISS), Daejeon 34113, Korea \\ * Correspondence: cnamki@jnu.ac.kr (N.C.); hmy@kriss.re.kr (H.M.Y.); Tel.: +82-62-530-2925 (N.C.); \\ +82-42-868-5362 (H.M.Y.) \\ + These authors contributed equally to this paper.
}

Academic Editors: Wolfgang Kreis and Jennifer Munkert

Received: 24 July 2019; Accepted: 15 August 2019; Published: 15 August 2019

check for updates

\begin{abstract}
Betula platyphylla bark has been evaluated for the treatment of dermatitis, inflammatory conditions, and cancer. Diarylheptanoids are the major constituents of the B. platyphylla bark and possess various pharmacological effects. Our previous study confirmed the selective antiproliferative effect of platyphylloside (BPP) isolated from B. platyphylla on colon cancer and leukemic cells using 60 different cancer cell lines from thr National Cancer Institution (NCI). In line with previous reports, this study focuses on the apoptotic pathway of BPP, a phenolic glycoside composed of two aromatic rings joined by a seven-carbon chain. Cytotoxicity assays in solid tumor and blood cancer cell models demonstrated that BPP possesses potent antiproliferative activity. The level of apoptosis increased with BPP treatment, causing cell cycle arrest at the G1 phase along with the downregulation of $\mathrm{I} \kappa \mathrm{B} \alpha$ phosphorylation and BCL-2, as well as upregulation of cleaved caspase 3 and BAX proteins. In addition, BPP displayed potent mitochondrial depolarization effects in Jurkat cells. The combined findings revealed that the cytotoxic effects of BPP were mediated by intracellular signaling, possibly through a mechanism involving the upregulation of mitochondrial reactive oxygen species (ROS). Thus, BPP could be a potential multitarget therapeutic agent in leukemia and colon cancer.
\end{abstract}

Keywords: platyphylloside; Betula platyphylla; apoptosis; reactive oxygen species (ROS); Jurkat cells

\section{Introduction}

Cancer is the second leading cause of death in the United States, and colorectal cancer (CRC) is one of the most common cancers in both men and women worldwide [1]. In Korea, cancer headed the list of the top 10 leading causes of death, and colorectal cancer (CRC) is the third most common cancer in men and women, with more than one million cases diagnosed each year worldwide [2]. Chemotherapeutic regimens, including 5-fluorouracil (5-FU), oxaliplatin, and irinotecan, are reportedly efficacious in colon cancer [3]. Additional targeted agents, including anti-EGFR or anti-VEGF antibodies, are considered to be effective in metastatic CRC cases (mCRC) [3]. Recently, research efforts in CRC drug discovery have focused on compounds derived from natural products due to the limited associated side effects [4]. Leukemia is a type of blood cancer that usually begins in the bone marrow and results in high abnormal blood cell counts. It is categorized according to how quickly the disease develops (acute or chronic) and the blood cell type affected (lymphocytes or myelocytes). The four main leukemia types include acute lymphocytic leukemia or acute lymphoblastic leukemia (ALL), chronic lymphocytic leukemia (CLL), acute myelocytic leukemia (AML), and chronic myelocytic leukemia or 
chronic myelogenous leukemia (CML). Leukemia treatments include chemotherapy, radiation and targeted therapies, and stem cell transplants. However, the conventional therapies are limited by the associated high costs and anticancer drug toxicity, challenging researchers to develop biocompatible and cost-effective new drugs [5-7]. Recent findings have demonstrated the considerable potential of alternative interventions, such as immunotherapy and natural products (NPs), in the treatment of leukemia [5-7]. NPs are capable of targeting multiple cancers and may provide a more robust treatment of cancer by limiting treatment-acquired resistance, increasing the efficacy of individual components in a cancer therapy cocktail, and reducing the associated side-effects to achieve a positive treatment response [5-7].

A platyphylloside denoted as BPP (Betula platyphylla platyphylloside) is a well-known diarylheptanoid obtained from the bark of Betula platyphylla (birch tree), which is widely distributed in Korea, Japan, China, Sahalin island, and Siberia [8]. The healing properties of B. platyphylla bark have long been known in traditional medicine located in different parts of the world [8,9]. Its most widespread uses have been in inflammatory diseases, including dermatitis, bronchitis, and cancer treatment $[8,9]$. B. platyphylla diarylheptanoids are phenolic compounds composed of two aromatic rings joined by a seven-carbon chain, containing various phenylpropanoid pathway-derived substituents. These compounds exhibit a wide range of biological activities, including antioxidant, anti-inflammatory, and anticancer activities [10-19]. Two major B. platyphylla diarylheptanoids, previously isolated with an efficient high speed counter current chromatography (HSCCC) method, showed antiproliferative potential in 60 National Cancer Institute (NCI) cancer cell lines [20]. Our previous study reported that platyphylloside—one of the two major B. platyphylla bark diarylheptanoids [19]—showed potent cytotoxic activities against several cancer cell lines, with selectively towards colon cancer and leukemic cells [20]. However, BPP's mechanism of action in colon and leukemia cell lines has not been established to date. Herein, BPP's antiproliferative and cell cycle effects on colorectal and blood cancer cells were determined. Furthermore, BPP-induced apoptosis was studied to understand its antiproliferative mechanism of action.

\section{Results and Discussion}

RKO, the human colorectal cancer cell line, was used to examine the antitumor effect of BPP [21]. Furthermore, human leukemic Jurkat T-cells were used to assess the antiproliferative and proapoptotic activity of BPP [22]. In this study, we employed solid tumor and blood cancer cell lines to evaluate the potential of BPP as a multitarget therapeutic agent, in both colon cancer and leukemia. To evaluate the mechanism of BPP in vitro, a large amount of BPP (Figure 1A) was isolated from the $\mathrm{CH}_{2} \mathrm{Cl}_{2}$ fraction of the B. platyphylla bark extract using high-speed counter-current chromatography, as previously described [20]. In terms of the structure-activity relationships, in vitro results from previous studies suggest that the structure of BPP, which consist of two aromatic rings joined by a seven carbon chain along with a glucosyl group at C-5 and a carbonyl group at C-3, positively correlated with anticancer activities [20,23].

\subsection{BPP-Induced Antiproliferative Effects in Colon Cancer Cells}

To determine the antiproliferative potential of BPP, it was screened against multiple colon cancer cell lines (Figure S1), using a cell proliferation assay (CellTiter Glo). BPP showed a pronounced antiproliferative activity against RKO. The antiproliferative effect increased with the dose $(20 \mu \mathrm{M})$ and treatment duration of $48 \mathrm{~h}$ (Figure 1B). After BPP $(20 \mu \mathrm{M})$ treatment, cell viability significantly reduced. Microscopic analysis also showed that BPP treatment induced morphological changes compared to the dimethyl sulfoxide (DMSO) treatment (Figure 1C). Considering that BPP showed a slightly stronger cytotoxicity against RKO than the other multiple colon cancer cell lines (Figure S1), our subsequent studies focused on the mechanism of action of BPP in RKO. 


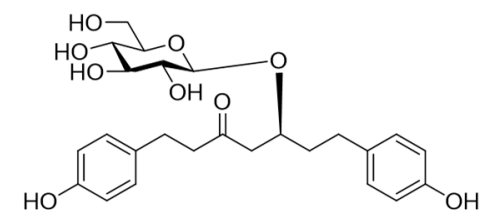

B

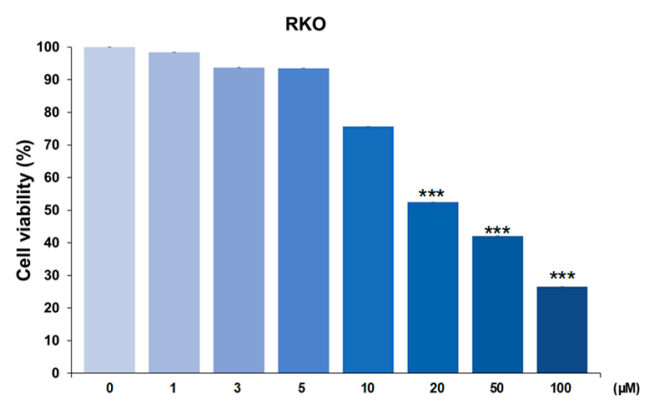

C

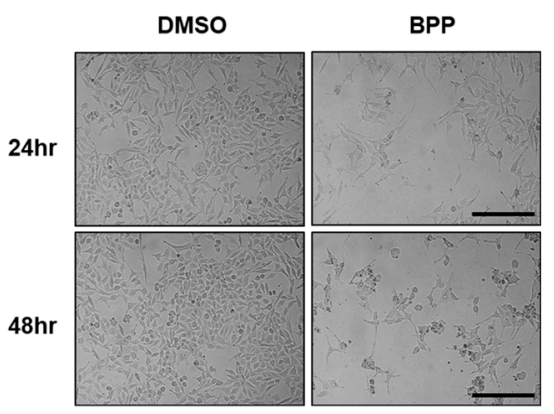

Figure 1. (A) Chemical structure of BPP (Betula platyphylla platyphylloside). (B) BPP showed an antiproliferative effect on RKO cells. Cell viability was determined using the MTS (3-(4,5-dimethylthiazol-2-yl)-5-(3-carboxymethoxyphenyl)-2-(4-sulfophenyl)-2H-tetrazolium, inner salt) assay $48 \mathrm{~h}$ post treatment with the compound. (C) The BPP $(20 \mu \mathrm{M})$-treated RKO (human colorectal cancer cell line) cells showed morphological changes characteristic of apoptosis. Phase contrast microscopy images (400× magnification) showing morphological changes 24 and $48 \mathrm{~h}$ post compound treatment.

\subsection{BPP-Induced Apoptosis in RKO Cells}

Moreover, BPP-induced apoptosis was determined using the RKO cell line. RKO cells were double-stained with annexin $\mathrm{V} / 7$-AAD dyes to determine the early and late apoptotic and viable cell percentages. The percentages of early $\left(\right.$ Annexin $\left.{ }^{+} / 7-\mathrm{AAD}^{-}\right)$and late apoptosis (Annexin $\left.{ }^{+} / 7-\mathrm{AAD}^{+}\right)$cells increased and the live cells decreased, $24 \mathrm{~h}$ post BPP treatment, confirming the induction of apoptosis (Figure 2A,B). BPP-treated RKO cells showed a dramatic increase in early (43.3\%) apoptotic cell numbers within $48 \mathrm{~h}$ of treatment (Figure 2A). Apoptosis-related protein levels in RKO cells in response to BPP $(20 \mu \mathrm{M})$ treatment were also analyzed using western blots (WB). We found that BPP downregulated the antiapoptotic protein BCL2, upregulated BAX, and cleaved caspase 3 expression (Figure 2C). To define more molecular mechanisms underlying BPP-induced cell apoptosis, we investigated NF- $\mathrm{KB}$ signaling by evaluating the phosphorylation of $\mathrm{I} \kappa \mathrm{B} \alpha$ protein. NF- $\mathrm{kB}$ is a critical transcription factor that regulates many genes associated with tumorigenesis [24]. Our data indicate that the phosphorylation of the inhibitory protein, $\mathrm{I} \kappa \mathrm{B} \alpha$, was reduced by BPP (Figure 2D). 
A

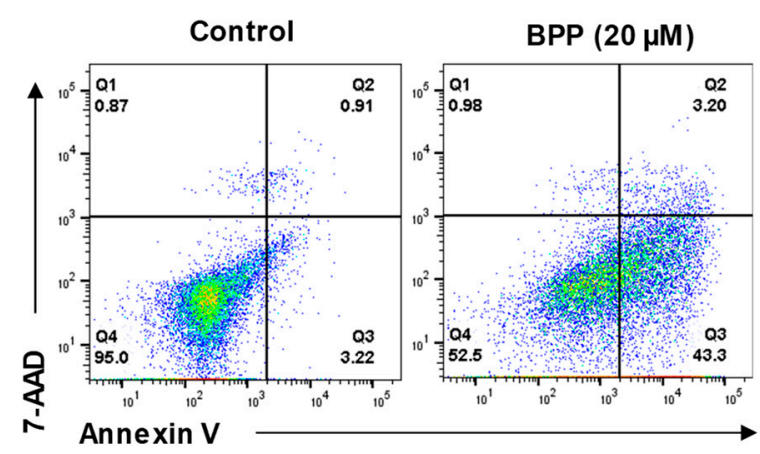

B

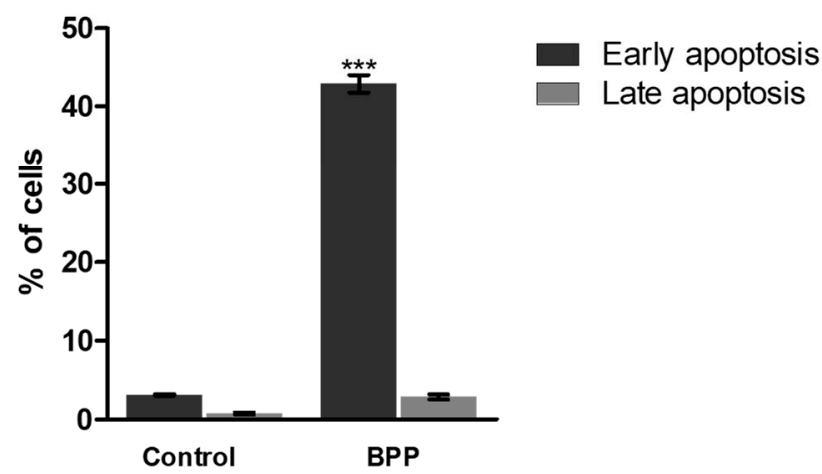

C

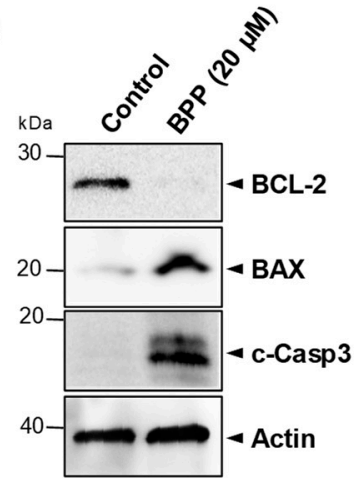

D

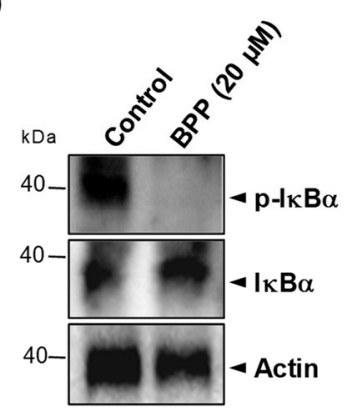

Figure 2. BPP-induced apoptosis in RKO cells. (A) RKO cells were compound-untreated or -treated, and apoptosis was evaluated $48 \mathrm{~h}$ later using annexin V/7AAD double staining. Cell death was assessed by flow cytometry. (B) Quantification of the early and late apoptotic cell rates. (C) Whole cell lysates from BPP-treated cells were immunoblotted with antibodies specific for BCL-2, BAX, and caspase-3 proteins. (D) Whole cell lysates were used to determine the expression levels of I-kappa-B-alpha (IkB $\alpha$ ) and $\mathrm{p}-\mathrm{I \kappa B} \alpha$ after treating the cells with BPP $(20 \mu \mathrm{M})$.

\subsection{BPP-Induced Cell Cycle Arrest in RKO Cells}

The inhibition of cell viability by BPP, due to effects on cell cycle progression, was investigated in RKO cells (Figure 3A,B). RKO incubation with BPP resulted in an increased sub-G1 and decreased S phase cell percentages. Similar results have been reported in colon cancer cells treated with curcumin, a diarylheptanoid isolated from Curcuma longa [25]. Previous in vitro studies suggest that, compared with diarylheptanoids, the coexistence of a glucosyl group at C-5 and a carbonyl group at C-3 in these diarylheptanoids is vital, and confers significant activities and selectivity towards cancer cells [23]. Our results also prove that BPP inhibits colon cancer cell growth by inducing cell cycle arrest in RKO cells. 
A

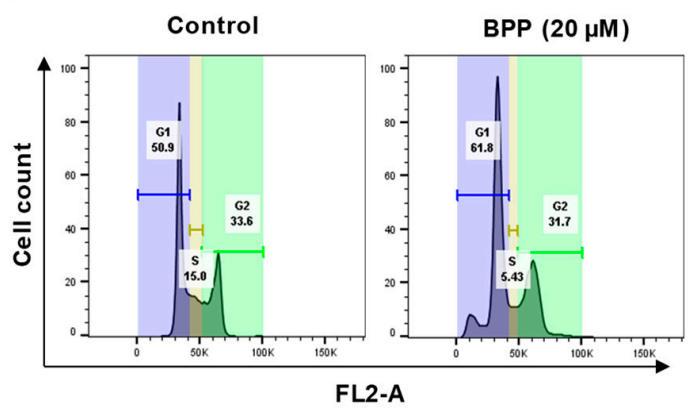

B

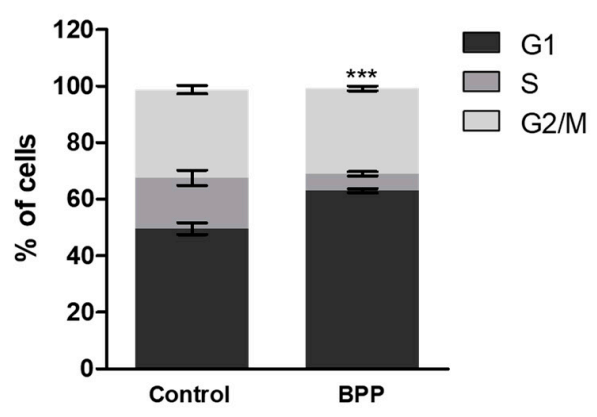

Figure 3. BPP-induced changes in RKO cell cycle progression. (A) Cell cycle analysis of compound-treated cells. Cells were stained with PI (propidium iodide) for $30 \mathrm{~min}$ and analyzed by flow cytometry. (B) Percentage G1 or S, G2/M phase cells from triplicate experiments were determined.

\subsection{BPP-Induced Antiproliferative Effects in Leukemic Cells}

The antiproliferative activity of BPP has been reported in solid tumors such as colorectal cancer $(\mathrm{CRC})$, but the effect is largely unexplored in blood cancers such as leukemia. This study, to our knowledge, is the first to evaluate the anticancer effect of BPP in blood cancer using Jurkat leukemia cell lines. To further assess the growth inhibition properties of BPP, a dose-dependent MTS screening assay was performed in multiple blood cancer cell lines (Figure 4A, Figure S2). Dose-dependent growth inhibition was observed in each cell line, with the effect being notably stronger in Jurkat cells. Compared to dimethyl sulfoxide (DMSO) treatment, BPP treatment induced morphological changes. BPP treatment significantly reduced viability of Jurkat leukemia cells, but DMSO-pretreatment markedly reversed these cytotoxic effects (Figure 4B).

A

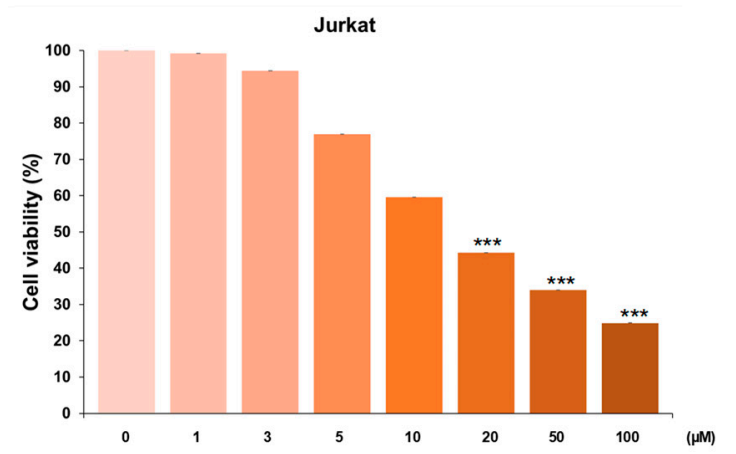

B

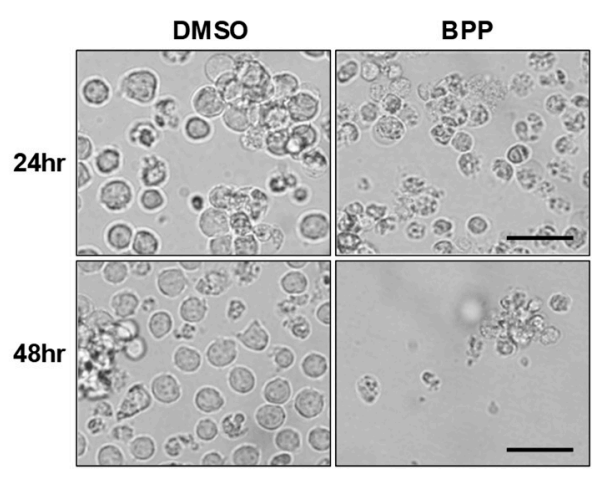

Figure 4. (A) BPP-induced antiproliferative effects on Jurkat cells. Cell viability was determined using an MTS assay $48 \mathrm{~h}$ post treatment. (B) The BPP $(20 \mu \mathrm{M})$-treated Jurkat cells showed morphological changes characteristic of apoptosis. Phase contrast microscopy images (400× magnification) showing morphological changes 24 and $48 \mathrm{~h}$ post treatment. Live cells are round in shape.

\subsection{BPP-Induced Apoptosis in Jurkat Cells}

To examine BPP-induced cell death in relation to apoptosis, we used the annexin V/7-AAD double staining-based fluorescence-activated cell sorting analysis. BPP-treated Jurkat cells showed a dramatic increase in both early $(12.3 \%)$ and late $(72.5 \%)$ apoptotic cell numbers within $48 \mathrm{~h}$ of treatment (Figure 5A). However, this apoptosis was reversed in the DMSO control (Figure 5B). As the apoptotic cell population dramatically increased, we investigated the relative apoptosis-associated protein expression levels. Western blots revealed that BPP downregulated the expression of the antiapoptotic protein BCL-2, a survival factor in apoptosis regulation and upregulated cleavage of poly (ADP-ribose) 
polymerase (PARP), which promotes apoptosis by preventing DNA repair, and cleaved caspase 3 (Figure 5C). In addition, BPP induced cell apoptosis in Jurkat cells through the downregulation of $\mathrm{p}-\mathrm{I} \kappa \mathrm{B} \alpha$, which is related to NF- $\kappa \mathrm{B}$ signaling, a critical transcription factor that regulates many genes associated with tumorigenesis (Figure 5D).

A

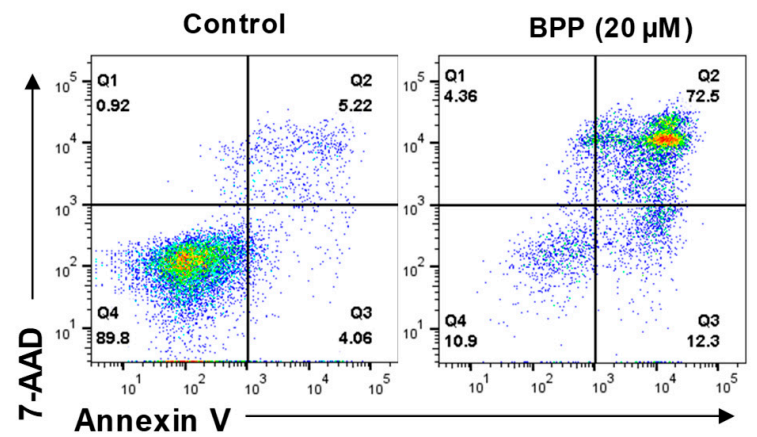

B

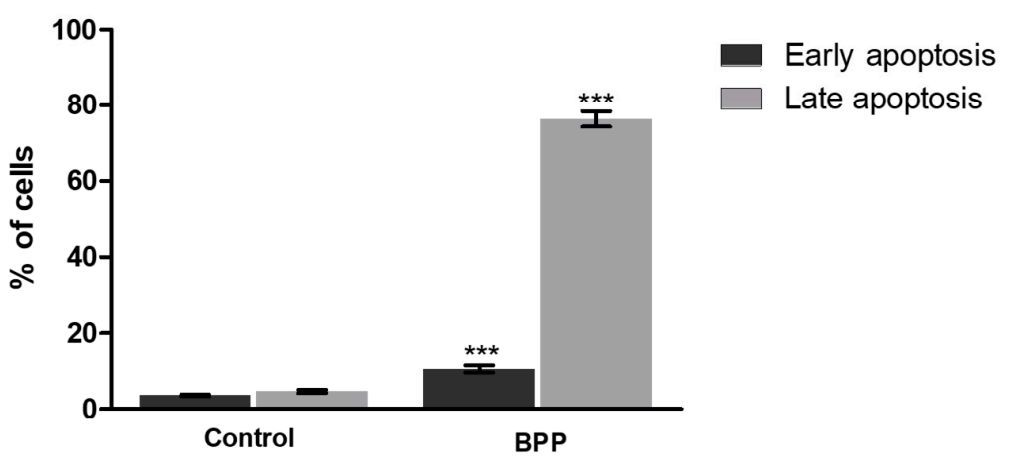

C

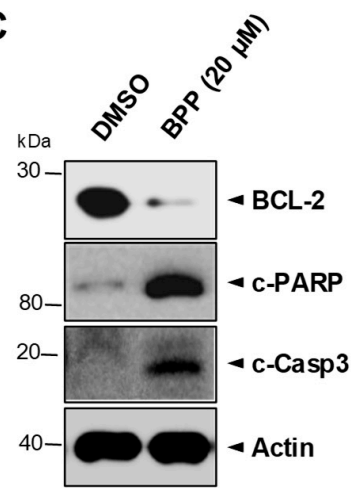

D

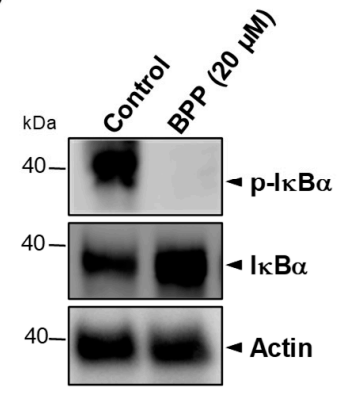

Figure 5. BPP-induced apoptosis in Jurkat cells. (A) Jurkat cells were compound-untreated or treated and apoptosis was evaluated $48 \mathrm{~h}$ thereafter using annexin V/7AAD double staining. Cell death was assessed by flow cytometry. (B) Early and late apoptotic cell rate quantification. (C) Whole cell lysates from BPP-treated cells were immunoblotted with antibodies specific for BCL-2, cleaved PARP, and cleaved caspase 3 proteins. (D) Whole cell lysates were used to determine the expression levels of I-kappa-B-alpha $(\mathrm{I} \kappa \mathrm{B} \alpha)$ and $\mathrm{p}-\mathrm{I} \kappa \mathrm{B} \alpha$ after treating cells with BPP $(20 \mu \mathrm{M})$.

\subsection{BPP-Induced Cell Cycle Arrest in Jurkat Cells}

We also found that BPP markedly increased the sub-G1 cell population. BPP $(20 \mu \mathrm{M})$ treatment for $48 \mathrm{~h}$ caused accumulation of cells in the sub-G1 phase, from $70.7 \%$ in the untreated control cells to $78.0 \%$ following BPP treatment (Figure 6A,B). A similar result was reported for Jurkat cells treated with curcumin [26]. Overall, these results of BPP-induced accumulation of apoptotic leukemia cell populations reveal that BPP may be responsible for the inhibition of cell growth via induction of cell cycle arrest at the sub-G1 phase. 
A

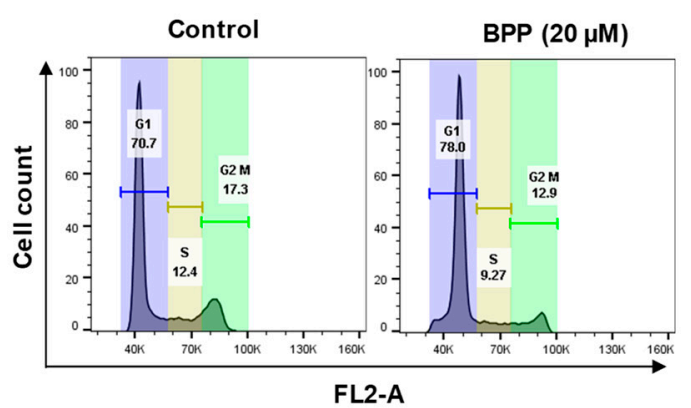

B

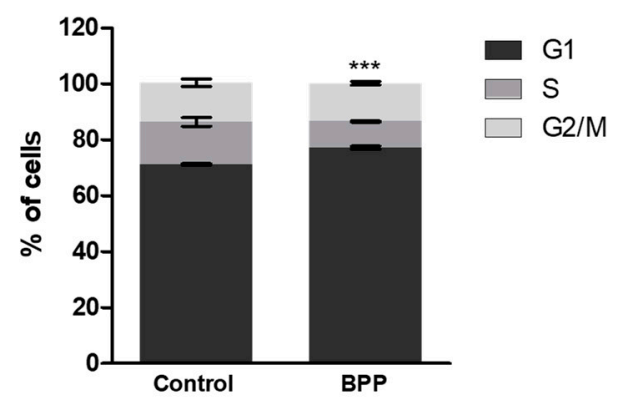

Figure 6. BPP-induced changes in cell cycle progression in Jurkat cells. (A) Cell cycle analysis of compound-treated cells. Cells were PI (propidium iodine)-stained for $30 \mathrm{~min}$ and analyzed by flow cytometry. (B) Percentage G1, S, G2/M phase cells were determined from triplicate experiments.

\subsection{BPP Effects on Mitochondrial Membrane Potential in Apoptotic Cells}

Next, BPP effects on the mitochondrial membrane potential $(\Delta \Psi \mathrm{m})$ were evaluated by probing tetramethylrhodamine methyl ester $\left(\mathrm{TMRM}^{+}\right)$fluorescence intensity using flow cytometry. $\mathrm{TMRM}^{+}$ quantifies changes in the live cell mitochondrial membrane potential. Figure 7A shows representative data from DMSO-treated negative controls and BPP-treated Jurkat cells. BPP-treated $(20 \mu \mathrm{M})$ cells showed significant membrane potential depolarization after $48 \mathrm{~h}$ (Figure 7B). Thus, BPP showed mitochondrial depolarization properties in Jurkat leukemic cells. These results suggest that B. platyphylla BPP could be a potential chemotherapeutic agent for blood cancer.

A

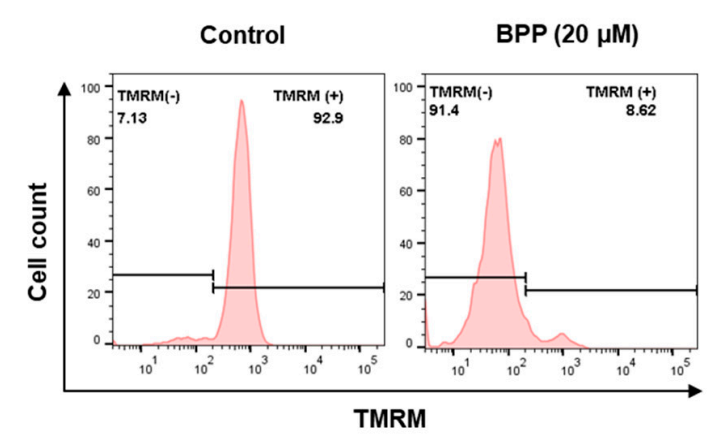

B

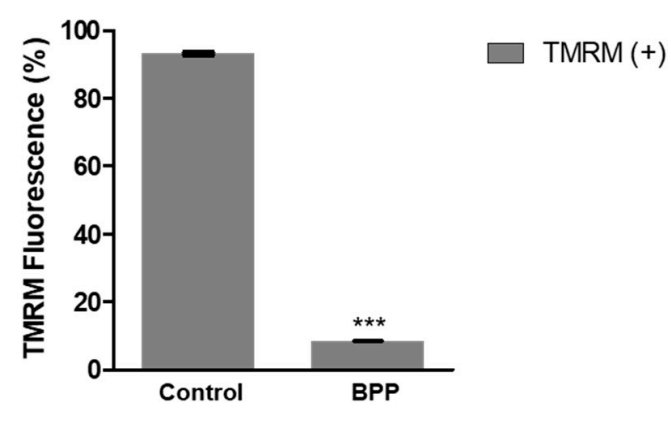

Figure 7. BPP-reduced Jurkat cell mitochondrial membrane potential. (A) Mitochondrial membrane potential of Jurkat cells loaded with TMRM $(100 \mathrm{nM})$ and detected by flow cytometry. (B) Quantification of mitochondrial membrane potential.

\subsection{BPP-Induced ROS in Jurkat Cells}

Mitochondria are major producers of reactive oxygen species (ROS) in cancer cells [27,28]. An elevated redox imbalance, relative to normal cells, is a common feature of cancer progression [29]. Association of ROS production with apoptosis and cell cycle arrest caused by anticancer agents has been demonstrated $[28,29]$. To examine how BPP causes cell death, ROS levels in each well were evaluated using the $2^{\prime}, 7^{\prime}$-dichlorodihydrofluorescein diacetate (H2DCFDA) assay.

Using confocal microscopy, we observed that BPP increased intracellular ROS levels in Jurkat cells after $48 \mathrm{~h}$ (Figure 8A). ROS generation was quantified by flow cytometry. Similar to the results observed by fluorescence microscopy, ROS generation in Jurkat cells was increased by BPP treatment (Figure 8B,C). Collectively, our results suggest that ROS generation by BPP is a potential mechanism underlying ROS-dependent apoptosis in Jurkat leukemic cells. 
A

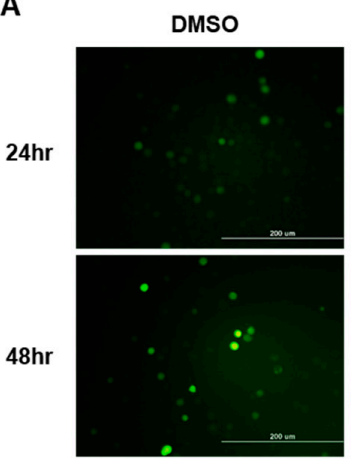

C

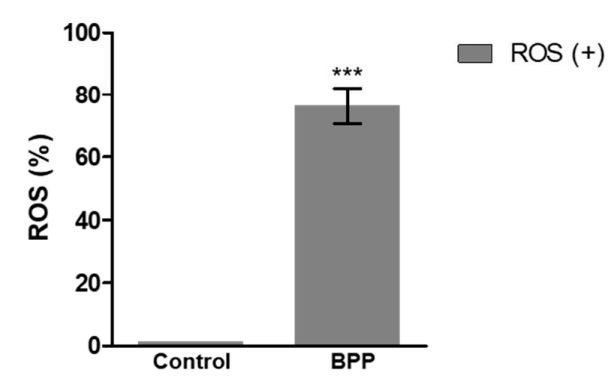

B

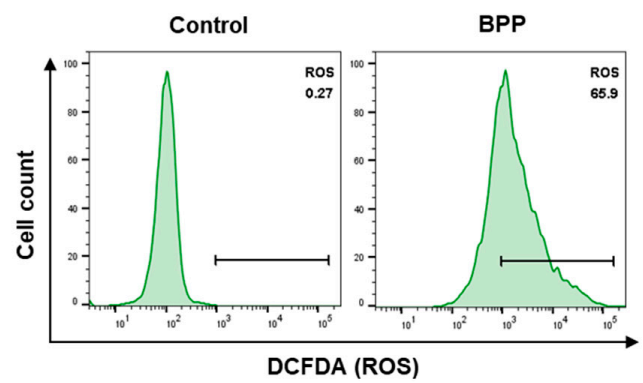

Figure 8. BPP-induced changes in intracellular ROS (reactive oxygen species) levels in Jurkat cells. (A) ROS detection by measurement of fluorescence intensity using fluorescence microscopy. (B) Jurkat cellular ROS levels were detected by flow cytometry with DCFDA $\left(2^{\prime}, 7^{\prime}\right.$-dichlorofluorescin diacetate $)$ $(1 \mu \mathrm{M})$. (C) Quantified ROS levels.

\subsection{Live/Dead Assay in Jurkat Cells}

The live/dead viability/cytotoxicity assay is most commonly used to validate and quantify live and dead cell populations, which involves labeling them with calcein (green) and ethidium homodimer-1 (red) fluorophores [30]. The live/dead assay was imaged by fluorescence microscopy (Figure 9A). As is typical of the live/dead stain, the BPP-exposed population demonstrated red fluorescence (white arrow) and weak green fluorescence. BPP-treated Jurkat cells showed a dramatic increase in red fluorescence-labeled dead cells within $48 \mathrm{~h}$ of treatment. However, this observation was reversed in the DMSO control (Figure 9B). These results corroborate the above results in which BPP significantly elevated the proportion of apoptotic cells in this study.

A

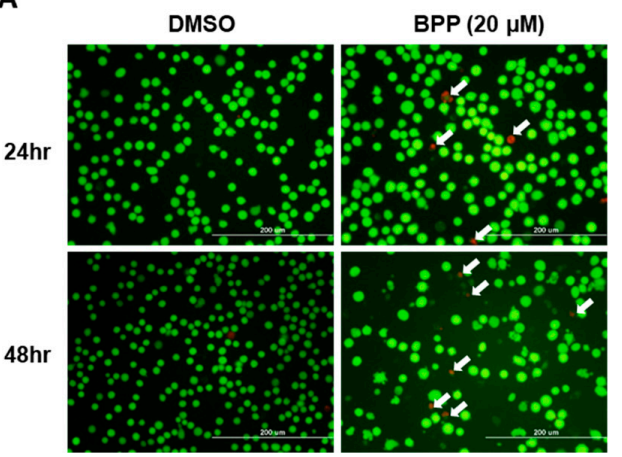

B

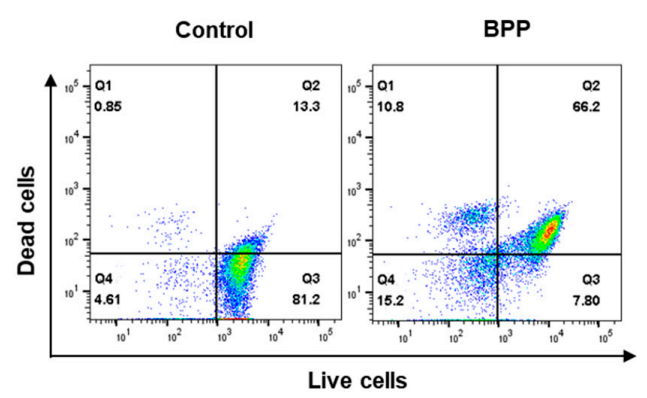

Figure 9. Measuring BPP-treated Jurkat cell viability. (A) Merged fluorescence images showing untreated and $48 \mathrm{~h}$ BPP-exposed Jurkat cells (green fluorescence, live cells; red fluorescence, dead cells). (B) Flow cytometry analysis of live and dead Jurkat cells with calcein and ethidium homodimer-1 staining. 


\section{Materials and Methods}

\subsection{Plant Material}

Betula platyphylla bark was obtained from the afforested land of SK E \& C (Seoul, Korea), and a voucher specimen (SNU-797) was deposited in the Herbarium of the Medicinal Plant Garden, College of Pharmacy, Seoul National University, Korea. High yield platyphylloside (5.0\%) was isolated from the Betula platyphylla $\mathrm{CH}_{2} \mathrm{Cl}_{2}$ fraction using high-speed counter-current chromatography, as previously descried [20].

\subsection{Cell Culture}

RKO, WiDr, HT-29, SW48, SW480, SW620, LoVo, HCT-116, DLD-1, LS174T, Jurkat, Daudi, and HEL 92.1.7 cells were obtained from the American Type Culture Collection (ATCC, Manassas, VA, USA). OCI-LY3 was obtained from The European Molecular biology laboratory (DSMZ, Braunschweig, Germany). The culture medium for each cell line was prepared according to the ATCC protocol. Cell lines were cultured in a humidified atmosphere of $5 \% \mathrm{CO}_{2}$ at $37^{\circ} \mathrm{C}$ and subcultured at a ratio of $1: 5$, when the cell density reached $80-90 \%$, every 3 or 4 days.

\subsection{Evaluating BPP-Induced Antiproliferation of Colon Cancer and Leukemic Cells}

The antiproliferative effect of BPP on colon and blood cancer cells was measured using the CellTiter 96 AQueous One Solution Cell Proliferation Assay Kit (Promega, Madison, WI, USA). The cells were cultured at a density of $5 \times 10^{5}$ cells/well in RPMI $1640(100 \mu \mathrm{L})$ for $24 \mathrm{~h}$. The cells were then treated with different BPP concentrations $(0 \mu \mathrm{M}, 1 \mu \mathrm{M}, 3 \mu \mathrm{M}, 5 \mu \mathrm{M}, 6 \mu \mathrm{M}, 10 \mu \mathrm{M}, 20 \mu \mathrm{M}, 50 \mu \mathrm{M}$, and $100 \mu \mathrm{M})$. DMSO-treated cells served as the control. To estimate cell viability (\%) $48 \mathrm{~h}$ post BPP treatment, the cells were incubated with $20 \mu \mathrm{L}$ of CellTiter 96 AQueous One Solution Cell Proliferation Assay reagent for 2 $\mathrm{h}$ at $37^{\circ} \mathrm{C}$. The optical density (OD) at $490 \mathrm{~nm}$ was measured using a microplate reader (Synergy HTX, BIO-TEK Instruments, Inc., Winooski, VT, USA).

\subsection{Microscopy}

BPP-treated RKO and Jurkat cells were examined under a phase contrast microscope (Olympus, Tokyo, Japan) to detect morphological changes. Photomicrographs of the leukemic cells were taken (at 400×) 24 and $48 \mathrm{~h}$ post BPP treatment, and the cells were analyzed for changes in shape, size, and number.

\subsection{Cytotoxicity Assay}

To explain the BPP-induced cytotoxicity mechanism in RKO and Jurkat cells, we investigated changes in cell cycle progression and apoptosis onset using flow cytometry.

\subsection{Determination of BPP-Induced Apoptosis}

BPP-treated RKO and Jurkat cells were visualized by flow cytometry using the PE Annexin V Apoptosis Detection Kit with 7-AAD (BioLegend, San Diego, CA, USA). Annexin V binds cell phosphatidylserine (PS) as PS flips from the inner to the outer leaflet of the plasma membrane during induction of early apoptosis induction. In contrast, PI stains DNA when the cell membrane is disrupted during late apoptosis. To distinguish PE from PI fluorescence and discriminate between early $\left(\right.$ Annexin $\mathrm{V}^{+}$) and late apoptotic cell $\left(7-\mathrm{AAD}^{+}\right)$populations, FL-2 and FL-3 channels were selected to measure the PE and 7-AAD fluorescence, respectively. BPP-treated and untreated (control) RKO and Jurkat cells were seeded in 6-well plates and incubated at $37^{\circ} \mathrm{C}$ for $48 \mathrm{~h}$. Cells were harvested 48 $h$ post-treatment, washed with phosphate-buffered saline (PBS), and resuspended in binding buffer $(1 \times)$. Cells were stained with annexin V/7-AAD, per the manufacturer's instructions. After staining, the cells were discriminated as early (Annexin $\mathrm{V}^{+} / 7-\mathrm{AAD}^{-}$) and late (Annexin $\mathrm{V}^{+} / 7-\mathrm{AAD}^{+}$) apoptotic 
cells using a flow cytometer (BD FACSVerse, BD Biosciences, San Jose, CA, USA). Cell percentages were analyzed using the FlowJo software (Becton, Dickinson and Company, Franklin Lakes, NJ, USA).

\subsection{Western Blot}

Cell lysates were prepared with lysis buffer containing $25 \mathrm{mM}$ of Tris- $\mathrm{HCl}$ (pH 7.6), $150 \mathrm{mM}$ of NaCl, $1 \%$ NP-40, 1\% sodium deoxycholate, and $0.1 \%$ sodium dodecyl sulfate, supplemented with a protease inhibitor cocktail (complete, Roche Molecular Biochemicals, Basel, Swtizerland). Lysates were placed on ice for $5 \mathrm{~min}$, and then centrifuged at $13,000 \mathrm{rpm}$ at $4{ }^{\circ} \mathrm{C}$ for $10 \mathrm{~min}$. Total protein concentrations were measured with Pierce BCA protein assay (ThermoFisher Scientific, Waltham, MA, USA). Total protein lysates (40 $\mu \mathrm{g}$ per lane) were separated by sodium dodecyl sulfate-polyacrylamide gel electrophoresis (SDS-PAGE) on $4-12 \%$ Bis Tris-NuPage polyacrylamide gels using $1 \times$ MES as running buffer (ThermoFisher Scientific, Waltham, MA, USA), and transferred to PVDF membranes $(0.45$ $\mu \mathrm{M}$ pore size,). After blocking nonspecific binding with $5 \%$ nonfat dry milk, membranes were probed with the following monoclonal or polyclonal primary antibodies, according to the manufacturer's recommendations: anti-cleaved PARP-1, anti-BCL-2, anti-BAX, I-kappa-B-alpha ( $\mathrm{I} \kappa \mathrm{B} \alpha)$, phosphorylated I-kappa-B-alpha ( $\mathrm{p}-\mathrm{I} \kappa \mathrm{B} \alpha)$, anti-actin (Santa Cruz Biotechnology, Inc., Dallas, TX, USA), and anti-cleaved caspase 3 (Cell signaling Techonology, Danvers, MA, USA). For detection, membranes were incubated with HRP-conjugated secondary antibodies (Jackson Laboratory, Bar Harbor, ME, USA), at 1:5000 dilution each, scanned with an ImageQuant LAS 4000 mini (Fujifilm, Tokyo, Japan), and analyzed with an image analysis program (Multi Gauge Ver. 3.0, Fujifilm, Tokyo, Japan).

\subsection{Tetramethylrhodamine Methyl Ester Perchlorate (TMRM) Assay}

BPP-treated Jurkat cells were harvested $48 \mathrm{~h}$ post-incubation. The cells were washed with PBS and incubated with $100 \mathrm{nM}$ cell-permeable fluorescent indicator TMRM (ThermoFisher Scientific, Waltham, MA, USA), prepared by diluting $10 \mathrm{mM}$ stock in PBS for $30 \mathrm{~min}$ at $37^{\circ} \mathrm{C}$. For mitochondrial membrane potential analysis, the cells were washed and resuspended in PBS, measured with a flow cytometer (BD FACSVerse, BD Biosciences, San Jose, CA, USA), and analyzed using the FlowJo v10 software (Becton, Dickinson and Company, Franklin Lakes, NJ, USA)

\subsection{Evaluating BPP-Induced Changes in Cell Cycle Progression}

Univariate analysis of the cellular DNA content of BPP-treated cells enables the detection of cells in different cycle phases (G0/G1, S, or G2/M) and apoptotic cells with fractional DNA content. The BPP-treated RKO and Jurkat cells were seeded into 6-well plates and incubated for $48 \mathrm{~h}$. The cells were harvested, washed with PBS, and fixed with ice-cold $70 \%$ ethanol for $3 \mathrm{~h}$ at $4{ }^{\circ} \mathrm{C}$. For cell cycle analysis, the cells were resuspended in PBS containing RNase A $(5 \mu \mathrm{g} / \mathrm{mL})$ and PI $(50 \mu \mathrm{g} / \mathrm{mL})$. The cells were evaluated using a flow cytometer (BD FACSVerse, BD Biosciences, San Jose, CA, USA) and cell percentages G0/G1, S, and G2/M phases were analyzed using the FlowJo software (Becton, Dickinson and Company, Franklin Lakes, NJ, USA).

\subsection{Measuring Reactive Oxygen Species (ROS)}

Intracellular ROS levels were detected using $2^{\prime}, 7^{\prime}$-dichlorodihydrofluorescein diacetate acetyl ester (H2DCFDA; Thermo Fisher Scientific, Waltham, MA, USA). Cells continuously perfused with PBS buffer $\left(37^{\circ} \mathrm{C}\right.$ ) were imaged using an Inverted fluorescent microscope (Olympus). For this analysis, cells were incubated with DCFDA $(1 \mu \mathrm{M})$ for $30 \mathrm{~min}$ at room temperature. Cells were also washed with cold PBS and resuspended in $0.5 \mathrm{~mL}$ of PBS supplemented with $1 \%$ fetal bovine serum. Intracellular fluorescence accumulation was analyzed using a flow cytometer (BD FACSVerse, BD Biosciences, San Jose, CA, USA). 


\subsection{Live/Dead Fluorescence Microscopy Assay}

Jurkat cell morphology was investigated by the simultaneous fluorescent labeling of both living and dead cells using the LIVE/DEAD kit (Thermo Fisher Scientific, Waltham, MA, USA). They were stained for $20 \mathrm{~min}$ in the dark using a calcein AM and ethidium homodimer (EthD-1), as per the manufacturer's instructions. Images were captured using a fluorescence microscope (Olympus, Tokyo, Japan).

\subsection{Statistical Analyses}

Statistical analyses were performed using GraphPad Prism 5 (GraphPad Software, Inc., San Diego, CA, USA), and the values are presented as the mean \pm S.D. The results were further analyzed by one-way ANOVA and t-test, $p$-values $<0.05$ were considered statistically significant.

\section{Conclusions}

Platyphyolloside (5-hydroxy-1,7-bis-(4-hydroxy-phenyl)-3-heptan-one-3-O- $\beta$-dglucopyranoside) is an arylheptanoid glucoside. Its mechanism of action in colon and leukemia cell lines has never been previously reported. In this study, we show that platyphylloside inhibits cell growth by inducing cell cycle arrest and apoptosis in both colorectal and leukemia cell lines, targeting more than one cancer-related mechanism to exert its antiproliferative activity. We investigated the cell cycle effect, and our results suggest that cell cycle arrest plays an important role in the inhibitory effect of BPP in RKO colon and Jurkat leukemia cells by preventing NF- $\mathrm{BB}$ signaling. Furthermore, platyphylloside induced mitochondrial depolarization and increased apoptosis in Jurkat cells. Furthermore, platyphylloside upregulated mitochondrial ROS to potentiate the cytotoxic effects via intracellular signaling. Although further studies are required to evaluate its in vivo anticancer activity, we were able to demonstrate that platyphylloside can be a potent multitarget anticancer chemotherapeutic agent by inducing cell cycle arrest and apoptosis via a ROS-dependent pathway.

Supplementary Materials: The following are available online, Figure S1: Anti-proliferative effects of BPP on various colon cancer cells. Figure S2: Anti-proliferative effects of BPP on various leukemia cells.

Author Contributions: N.C. and H.M.Y., conceived and designed the experiments; N.C., N.T.T.T., J.L., J.L., and H.M.Y., performed the experiments; N.T.T.T and J.L., wrote the manuscript; N.T.T.T., J.L., H.M.Y., and N.C., analyzed the data; H.M.Y and N.C., revised the paper.

Funding: This research was funded by a grant from the Development of New Chemical Medical Measurement Standard Technology, funded by Korea Research Institute of Standards and Science (KRISS-2019-GP2019-0009). This work was also supported by a grant from the National Research Foundation of Korea (NRF), funded by the Korean government (MSIT; grant number: NRF-2018R1C1B5083127).

Acknowledgments: We thank Ji Hyun Kim for assistance with experiments.

Conflicts of Interest: The authors declare no conflict of interest.

\section{References}

1. Kuipers, E.J.; Grady, W.M.; Lieberman, D.; Seufferlein, T.; Sung, J.J.; Boelens, P.G.; Van De Velde, C.J.H.; Watanabe, T. Colorectal cancer. Nat. Rev. Dis. Prim. 2015, 1, 15065. [CrossRef] [PubMed]

2. Jung, K.W.; Won, Y.J.; Kong, H.J.; Lee, E.S. Cancer statistics in Korea: Incidence, mortality, survival, and prevalence in 2015. Cancer Res. Treat. 2018, 50, 303-316. [CrossRef] [PubMed]

3. Miura, K.; Satoh, M.; Kinouchi, M.; Yamamoto, K.; Hasegawa, Y.; Kakugawa, Y.; Kawai, M.; Uchimi, K.; Aizawa, H.; Ohnuma, S.; et al. The use of natural products in colorectal cancer drug discovery. Expert Opin. Drug Discov. 2015, 10, 411-426. [CrossRef] [PubMed]

4. Wong, K.E.; Ngai, S.C.; Chan, K.G.; Lee, L.H.; Goh, B.H.; Chuah, L.H. Curcumin nanoformulations for colorectal cancer: A Review. Front. Pharmacol. 2019, 10, 152. [CrossRef] [PubMed]

5. Greaves, M. Leukaemia "firsts" in cancer research and treatment. Nat. Rev. Cancer 2016, 16, 163-172. [CrossRef] [PubMed] 
6. Hwang, D.; Kim, M.; Park, H.; Jeong, M.I.; Jung, W.; Kim, B. Natural Products and Acute Myeloid Leukemia: A Review Highlighting Mechanisms of Action. Nutrients 2019, 11, 1010. [CrossRef]

7. Taylor, W.F.; Moghadam, S.E.; Farimani, M.M.; Ebrahimi, S.N.; Tabefam, M.; Jabbarzadeh, E. A multi-targeting natural compound with growth inhibitory and anti-angiogenic properties re-sensitizes chemotherapy resistant cancer. PloS ONE 2019, 14, 1-17. [CrossRef] [PubMed]

8. Huh, J.E.; Baek, Y.H.; Kim, Y.J.; Lee, J.D.; Choi, D.Y.; Park, D.S. Protective effects of butanol fraction from Betula platyphyla var. japonica on cartilage alterations in a rabbit collagenase-induced osteoarthritis. J. Ethnopharmacol. 2009, 123, 515-521. [CrossRef]

9. Rastogi, S.; Pandey, M.M.; Rawat, A.K.S. Medicinal plants of the genus Betula-Traditional uses and a phytochemical-pharmacological review. J. Ethnopharmacol. 2015, 159, 62-83. [CrossRef]

10. Brand, S.; Hölscher, D.; Schierhorn, A.; Svatoš, A.; Schröder, J.; Schneider, B. A type III polyketide synthase from Wachendorfia thyrsiflora and its role in diarylheptanoid and phenylphenalenone biosynthesis. Planta 2006, 224, 413-428. [CrossRef]

11. Rajaganapathy, B.R.; Thirugnanam, K.; Shanmuganathan, M.V.; Singaravelu, A.; Subadhra, L.B. Molecular basis of the anti-inflammatory potential of a diarylheptanoid in murine macrophage RAW 264.7 cells. Adv. Biol. Chem. 2013, 3, 541-548. [CrossRef]

12. Dehelean, C.A.; Şoica, C.; Ledeţi, I.; Aluaş, M.; Zupko, I.; Găluşcan, A.; Munteanu, M.; Munteanu, M. Study of the betulin enriched birch bark extracts effects on human carcinoma cells and ear inflammation. Chem. Cent. J. 2012, 6, 137. [CrossRef] [PubMed]

13. Huh, J.E.; Hong, J.M.; Baek, Y.H.; Lee, J.D.; Choi, D.Y.; Park, D.S. Anti-inflammatory and anti-nociceptive effect of Betula platyphylla var. japonica in human interleukin-1 $\beta$-stimulated fibroblast-like synoviocytes and in experimental animal models. J. Ethnopharmacol. 2011, 135, 126-134. [CrossRef] [PubMed]

14. Ju, E.M.; Lee, S.E.; Hwang, H.J.; Kim, J.H. Antioxidant and anticancer activity of extract from Betula platyphylla var. japonica. Life Sci. 2004, 74, 1013-1026. [CrossRef] [PubMed]

15. Ghimire, B.K.; Tamang, J.P.; Yu, C.Y.; Jung, S.J.; Chung, I.M. Antioxidant, antimicrobial activity and inhibition of $\alpha$-glucosidase activity by Betula alnoides Buch. bark extract and their relationship with polyphenolic compounds concentration. Immunopharmacol. Immunotoxicol. 2012, 34, 824-831. [CrossRef] [PubMed]

16. Duraiswamy, B.; Satishkumar, M.N.; Gupta, S.; Rawat, M.; Porwal, O.; Murugan, R. Hepatoprotective activity of Betula Utilis bark on D-galactosamine induced hepatic insult. World J. Pharm. Pharma. Sci. 2012, 1, 456-471.

17. Germanò, M.P.; Cacciola, F.; Donato, P.; Dugo, P.; Certo, G.; D'Angelo, V.; Mondello, L.; Rapisarda, A. Betula pendula Roth leaves: Gastroprotective effects of an HPLC-fingerprinted methanolic extract. Nat. Prod. Res. 2013, 27, 1569-1575. [CrossRef]

18. Germanò, M.P.; Cacciola, F.; Donato, P.; Dugo, P.; Certo, G.; D'angelo, V.; Mondello, L.; Rapisarda, A. Betula pendula leaves: Polyphenolic characterization and potential innovative use in skin whitening products. Fitoterapia 2012, 83, 877-882. [CrossRef]

19. Lee, K.Y.; Jeong, E.J.; Huh, J.; Cho, N.; Kim, T.B.; Jeon, B.J.; Kim, S.H.; Kim, H.P.; Sung, S.H. Cognition-enhancing and neuroprotective activities of the standardized extract of Betula platyphylla bark and its major diarylheptanoids. Phytomedicine 2012, 19, 1315-1320. [CrossRef]

20. Cho, N.; Kim, H.Y.; Kim, T.B.; Ransom, T.T.; Beutler, J.A.; Sung, S.H. Preparative purification of anti-proliferative diarylheptanoids from Betula platyphylla by high-speed counter-current chromatography. Molecules 2016, 21, 700. [CrossRef]

21. Moos, P.J.; Edes, K.; Mullally, J.E.; Fitzpatrick, F.A. Curcumin impairs tumor suppressor p53 function in colon cancer cells. Carcinogenesis 2004, 25, 1611-1617. [CrossRef] [PubMed]

22. Uto, T.; Tung, N.H.; Appiah-Opong, R.; Aning, A.; Morinaga, O.; Edoh, D.; Nyarko, A.K.; Shoyama, Y. Antiproliferative and pro-apoptotic activity of diarylheptanoids isolated from the bark of Alnus japonica in human leukemia cell lines. Am. J. Chin. Med. 2015, 43, 757-767. [CrossRef] [PubMed]

23. Novaković, M.; Pešić, M.; Trifunović, S.; Vučković, I.; Todorović, N.; Podolski-Renić, A.; Dinić, J.; Stojković, S.; Tešević, V.; Vajs, V.; et al. Diarylheptanoids from the bark of black alder inhibit the growth of sensitive and multi-drug resistant non-small cell lung carcinoma cells. Phytochemistry 2014, 97, 46-54. [CrossRef] [PubMed]

24. Wu, Y.; Zhou, B.P. Inflammation: A driving force speeds cancer metastasis. Cell Cycle 2009, 8, 3267-3273. [CrossRef] [PubMed] 
25. Lim, T.G.; Lee, S.Y.; Huang, Z.; Chen, H.; Jung, S.K.; Bode, A.M.; Lee, K.W.; Dong, Z. Curcumin suppresses proliferation of colon cancer cells by targeting CDK2. Cancer Prev. Res. 2014, 7, 466-474. [CrossRef] [PubMed]

26. Gopal, P.K.; Paul, M.; Paul, S. Curcumin induces caspase mediated apoptosis in JURKAT cells by disrupting the redox balance. Asian Pac. J. Cancer Prev. 2014, 15, 93-100. [CrossRef] [PubMed]

27. Nickel, A.; Kohlhaas, M.; Maack, C. Mitochondrial reactive oxygen species production and elimination. J. Mol. Cell Cardiol. 2014, 73, 26-33. [CrossRef]

28. Sullivan, L.B.; Chandel, N.S. Mitochondrial reactive oxygen species and cancer. Cancer Metab. $2014,2,17$. [CrossRef]

29. Kumari, S.; Badana, A.K.; Murali Mohan, G.; Shailender, G.; Malla, R.R. Reactive Oxygen Species: A Key Constituent in Cancer Survival. Biomark. Insights 2018, 13, 1-9. [CrossRef]

30. Kummrow, A.; Frankowski, M.; Bock, N.; Werner, C.; Dziekan, T.; Neukammer, J. Quantitative assessment of cell viability based on flow cytometry and microscopy. Cytometry A 2013, 83, 197-204. [CrossRef]

Sample Availability: Samples of the compounds are available from the authors.

(C) 2019 by the authors. Licensee MDPI, Basel, Switzerland. This article is an open access article distributed under the terms and conditions of the Creative Commons Attribution (CC BY) license (http://creativecommons.org/licenses/by/4.0/). 